OPEN ACCESS

Edited by:

Muhammad Zubair, University of Engineering and Technology Taxila, Pakistan

Reviewed by:

Anca Melintescu,

Horia Hulubei National Institute of Physics and Nuclear Engineering,

Romania

Giuseppe Vella,

University of Palermo, Italy

Qazi Muhammad Nouman Amjad,

University of Engineering and

Technology Taxila, Pakistan

*Correspondence:

Satoshi Utsunomiya,

Department of Chemistry, Kyushu

University, Hakozaki 6-10-1,

Higashi-ku, Fukuoka, Japan utsunomiya.satoshi.998@

m.kyushu-u.ac.jp

Specialty section: This article was submitted to Nuclear

Energy, a section of the journal

Frontiers in Energy Research

Received: 22 June 2015

Accepted: 06 August 2015

Published: 01 September 2015

Citation:

Kaneko M, Iwata H, Shiotsu H, Masaki S, Kawamoto Y, Yamasaki S,

Nakamatsu Y, Imoto J, Furuki G,

Ochiai A, Nanba K, Ohnuki T,

Ewing RC and Utsunomiya S (2015)

Radioactive Cs in the severely

contaminated soils near the

Fukushima Daiichi nuclear power plant.

Front. Energy Res. 3:37.

doi: 10.3389/fenrg.2015.00037

\section{Radioactive Cs in the severely contaminated soils near the Fukushima Daiichi nuclear power plant}

\author{
Makoto Kaneko ${ }^{1}$, Hajime Iwata ${ }^{1}$, Hiroyuki Shiotsu ${ }^{1}$, Shota Masaki ${ }^{1}$, Yuji Kawamoto ${ }^{1}$, \\ Shinya Yamasaki ${ }^{1}$, Yuki Nakamatsu ${ }^{1}$, Junpei Imoto ${ }^{1}$, Genki Furuki ${ }^{1}$, Asumi Ochiai ${ }^{1}$, \\ Kenji Nanba ${ }^{2}$, Toshihiko Ohnuki ${ }^{3}$, Rodney C. Ewing ${ }^{4}$ and Satoshi Utsunomiya ${ }^{1 *}$
}

\begin{abstract}
'Department of Chemistry, Kyushu University, Fukuoka, Japan, ${ }^{2}$ Department of Environmental Management, Faculty of Symbiotic System Science, Fukushima University, Fukushima, Japan, ${ }^{3}$ Advanced Science Research Center Japan Atomic Energy Agency, Tokai, Japan, ${ }^{4}$ Department of Geological Sciences, Center for International Security and Cooperation, Stanford University, Stanford, CA, USA
\end{abstract}

Radioactive Cs isotopes ( ${ }^{137} \mathrm{Cs}, t_{1 / 2}=30.07$ years and ${ }^{134} \mathrm{Cs}, t_{1 / 2}=2.062$ years $)$ occur in severely contaminated soils within a few kilometer of the Fukushima Daiichi nuclear power plant at concentrations that range from $4 \times 10^{5}$ to $5 \times 10^{7} \mathrm{~Bq} / \mathrm{kg}$. In order to understand the mobility of $\mathrm{Cs}$ in these soils, both bulk and submicron-sized particles elutriated from four surface soils have been investigated using a variety of analytical techniques, including powder X-ray diffraction analysis, scanning electron microscopy (SEM), transmission electron microscopy (TEM), and analysis of the amount of radioactivity in sequential chemical extractions. Major minerals in bulk soil samples were quartz, feldspar, and minor clays. The submicron-sized particles elutriated from the same soil consist mainly of mica, vermiculite, and smectite and occasional gibbsite. Autoradiography in conjunction with SEM analysis confirmed the association of radioactive Cs mainly with the submicronsized particles. Up to $\sim 3 \mathrm{MBq} / \mathrm{kg}$ of ${ }^{137} \mathrm{Cs}$ are associated with the colloidal size fraction $(<1 \mu \mathrm{m})$, which accounts for $\sim 78 \%$ of the total radioactivity. Sequential extraction of the bulk sample revealed that most Cs was retained in the residual fraction, confirming the high binding affinity of Cs to clays, aluminosilicate sheet structures. The chemistry of the fraction containing submicron-sized particles from the same bulk sample showed a similar distribution to that of the bulk sample, again confirming that the Cs is predominantly adsorbed onto submicron-sized sheet aluminosilicates, even in the bulk soil samples. Despite the very small particle size, aggregation of the particles prevents migration in the vertical direction, resulting in the retention of $>98 \%$ of Cs within top $\sim 5 \mathrm{~cm}$ of the soil. These results suggest that the mobility of the aggregates of submicron-sized sheet aluminosilicate in the surface environment is a key factor controlling the current Cs migration in Fukushima.

Keywords: cesium, colloid, electron microscopy, Fukushima Daiichi nuclear disaster, clay minerals 


\section{Introduction}

Four years have passed since the Fukushima Daiichi nuclear power plant (FDNPP) event released $\sim 520$ PBq of radionuclides (Steinhauser et al., 2014) in the vicinity of FDNPP. An elongated area some $\sim 27$ miles in length and $\sim 7$ miles wide to the northwest of FDNPP is still contaminated with radioactivity, mainly ${ }^{134} \mathrm{Cs}$ and ${ }^{137} \mathrm{Cs}$, with half-lives of 2.06 and 30.07 years, respectively. A recent study reported an $\sim 40 \%$ decrease of radioactivity in this area (MEXT, 2013), which is greater than the decrease $(\sim 21 \%)$ that is expected from the decay constants for Cs, especially ${ }^{134} \mathrm{Cs}$. A number of studies have reported geochemical behavior of Cs in the contaminated area [e.g., Koarashi et al. (2012) and Yoshida and Takahashi (2012)] in order to understand the distribution and migration of radioactive Cs in the future. After the Cs release to atmosphere, most Cs aerosols were deposited by precipitation on March 15 (wet deposition), which continued through March 16th (Kinoshita et al., 2011; Kaneyasu et al., 2012; Morino et al., 2013) based on the chemical transport model, which were in good agreement with the actual Cs distribution. In the contaminated Fukushima soils, the Cs remains within the top $\sim 5 \mathrm{~cm}$ of the soil profile, strongly bound to clay minerals, probably vermiculite, and illite (Tanaka et al., 2012a). On the other hand, another study reported that other mineral component were also associated with irreversibly bound radioactive Cs, based on results obtained by chemical extraction and subsequent X-ray diffraction (XRD) analysis (Kozai et al., 2012; Ohnuki and Kozai, 2013). In addition, the presence of organic matter in soils may prevent the Cs adsorption onto mineral surfaces (Matsunaga et al., 2013).

Mukai et al. (2014) recently conducted transmission electron microscopy (TEM) analysis of Fukushima soils and showed that most particles associated with radioactive Cs are aggregates of clays and other phases, such as organic matter. This aggregation makes it difficult to characterize the submicron phases associated with Cs or to estimate their contribution to the total Cs radioactivity. It is well-known that submicron-sized particles behave as colloids in surface and subsurface aquifers (McCarthy and Zachara, 1989); thus, these colloids can potentially control the migration of trace radionuclides. Hence, we have completed a detailed analysis of the colloid sized and smaller fraction of the soils. Although the previous studies reported a high affinity of Cs with clay minerals in the Fukushima surface environments (Tanaka et al., 2012a,b; Matsunaga et al., 2013; Nakao et al., 2014), the role of fine particles $<1 \mu \mathrm{m}$ has not been quantitatively determined, which is presumably the size fraction dominated by clay minerals. Finally, the severely contaminated area close to FDNPP is not easily accessible, and thus, fewer data have been reported for the soil from this area as compared with data recently reported for the samples more than several kilometers from the FDNPP. The present study investigated that the soil particles collected within a few kilometer of FDNPP utilizing a variety of analytical techniques including conventional sequential extraction methods and electron microscopy in order to describe the mineralogy, identify the Cs speciation, and determine the dose contribution from Cs sorbed onto submicron-sized particles.

\section{Materials and Methods}

\section{Sample Description}

Samples were collected on March 15-16, 2012. The localities of the sample sites are shown in Figure 1. Four soil samples were collected in the highly contaminated area in Ohkuma within $5 \mathrm{~km}$ of the FDNPP: two samples from the east and west zones of the Ottozawa district and the other two samples from east and west zones of the Koirino district. Hereafter, these samples are identified as OTO-E, OTO-W, KOI-E, and KOI-W, respectively. The collected samples were dried and sieved with a 2-mm polyethylene mesh filter to remove pebbles and plant material, such as grass.

A soil sample core $(30 \mathrm{~cm}$ in length $\times 55 \mathrm{~mm}$ in diameter $)$ was also collected from KOI-E, using a DAIKI core sampler, DIK$110 \mathrm{C}$. The core was initially used to obtain the vertical crosssectional autoradiography in order to image the distribution of radionuclides as a function of depth. Then, the outermost part of this cylindrical core was carefully shaved off to exclude the contamination from the core boring process. The depth profile of ${ }^{134} \mathrm{Cs}$ and ${ }^{137} \mathrm{Cs}$ radioactivities was made at $1 \mathrm{~cm}$ intervals for

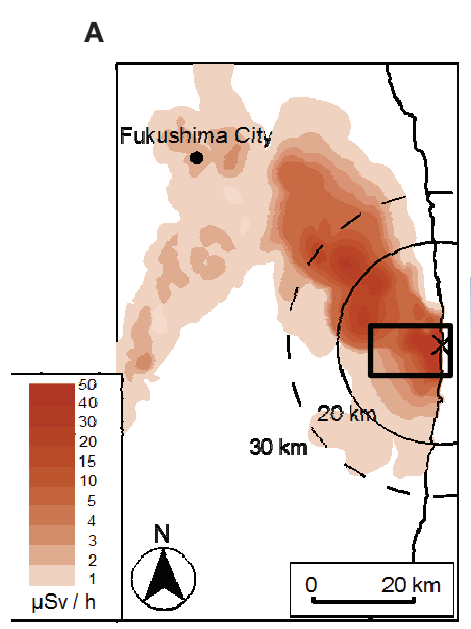

\section{B}

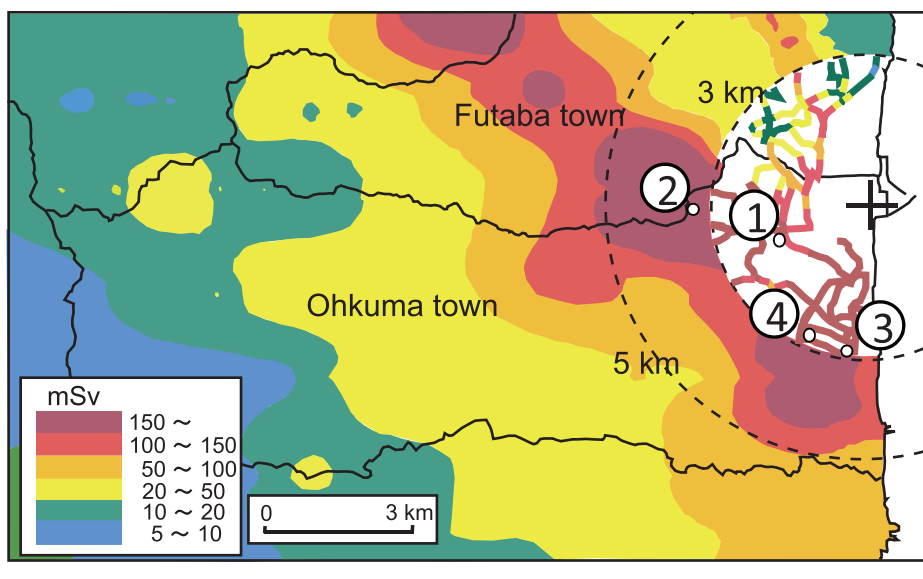

FIGURE 1 | Map of dose at $1 \mathbf{m}$ above the ground. Data from Ministry of Education, Culture, Sports, Science and Technology, Japan (MEXT, 2013). (A) Prediction of the accumulated dose during 1 year; 3/11/2011-2/11/2012. (B) Sampling location collected in March 2012. 
the core. The core sample was divided into 12 fractions by the depth: $0-1,1-2,2-3,3-4,4-5,5-6,6-7,7-8,8-9,9-10,10-13$, and $13-16 \mathrm{~cm}$.

\section{Analytical Methods Size Fractionation}

The size-dependent ${ }^{134,137} \mathrm{Cs}$ activity was determined for three samples: OTO-E, OTO-W, and KOI-E. The size separation was performed with a sequence of size fractions: $>597,114-597$, $48-114,1-48$, and $<1 \mu \mathrm{m}$. The soil was suspended in ultra-pure water (milli-Q) and ultrasonicated for $15 \mathrm{~min}$ and filtered first with a $597 \mu \mathrm{m}$ mesh. The residue was re-suspended in pure water, ultrasonicated, and filtered using a $114-\mu \mathrm{m}$ mesh. The size ranges, $>597,114-597$ and $48-114 \mu \mathrm{m}$, were obtained in the same manner. The soil particles $<1 \mu \mathrm{m}$ were separated from the $<48 \mu \mathrm{m}$ fraction by a sedimentation method, in which the hydrodynamic size was calculated based on the Stokes' law. The separated soils were air dried and weighed prior to further analysis. For the KOIE sample, the size-fractionated samples for the $>597,114-597$, and $48-114 \mu \mathrm{m}$ fractions were subsequently ultrasonicated twice for $30 \mathrm{~min}$ in order to separate fine particles that were attached to

TABLE $1 \mid{ }^{134}$ Cs and ${ }^{137}$ Cs radioactivities of the four bulk soils

\begin{tabular}{|c|c|c|}
\hline & \multicolumn{2}{|c|}{ Radioactivity (Bq/kg) } \\
\hline & ${ }^{134} \mathrm{Cs}$ & ${ }^{137}$ Cs \\
\hline (1) OTO-E & $2.71 \times 10^{5}$ & $3.80 \times 10^{5}$ \\
\hline (2) OTO-W & $8.37 \times 10^{5}$ & $1.11 \times 10^{6}$ \\
\hline (3) $\mathrm{KOl-E}$ & $1.85 \times 10^{5}$ & $2.50 \times 10^{5}$ \\
\hline (4) $\mathrm{KOI}-\mathrm{W}$ & $2.03 \times 10^{7}$ & $2.66 \times 10^{7}$ \\
\hline
\end{tabular}

large-sized particles and to quantify the actual contribution of the fine particles to the total radioactivity.

\section{Sequential Extraction of Radioactive Cs from Soils}

Sequential extraction was performed for OTO-E, OTO-W, and KOI-E samples. The extraction method was based on Hou et al. (2003), which is a modified procedure after Tessier et al. (1979). Using this method, the chemical form of the Cs associated with soils is separated into six chemical categories: water soluble (F1), exchangeable (F2), bound to carbonate (F3), bound to metal oxide (F4), bound to organic matter (F5), and the residue (F6). Although the details of the extraction procedure were described in Hou et al. (2003), the procedure performed in the present study is briefly summarized as follows; first, pure water was added to 5 or $10 \mathrm{~g}$ soil and the suspension was continuously agitated for $24 \mathrm{~h}$ at room temperature. Then, the solution was centrifuged at $5000 \mathrm{rpm}$ for $10 \mathrm{~min}$, and the supernatant was filtered with $0.025 \mu \mathrm{m}$ membrane filter. The filtrate was classified as water-soluble fraction (F1). The residue (residue-1) obtained by the filtering was re-suspended in $1.0 \mathrm{M} \mathrm{NH}_{4} \mathrm{OAc}(\mathrm{pH} 8.0$ ) and agitated for $24 \mathrm{~h}$ at room temperature. After the treatment, the suspension was centrifuged and filtered. The residue (residue-2) was rinsed with pure water and the rinsing water was added to the leachate. The filtrate was labeled as exchangeable (F2). Subsequently, the residue-2 was resuspended in $1.0 \mathrm{M} \mathrm{NH} \mathrm{NHAc}_{4} \mathrm{OH} \mathrm{5.0)}$ and agitated for $24 \mathrm{~h}$ at room temperature. The suspension was centrifuged and filtered. The residue (residue-3) was rinsed with pure water. The filtrate was labeled as the form bound to carbonate (F3). For the form bound to metal oxides (F4), $0.04 \mathrm{M} \mathrm{NH} \mathrm{NH}_{2} \mathrm{OH} \cdot \mathrm{HCl}$ in $25 \%(\mathrm{v} / \mathrm{v})$ $\mathrm{HOAc}$ at final $\mathrm{pH}$ of 2.0 was added to the residue-3, and the suspension was agitated for $24 \mathrm{~h}$ at $95 \pm 5^{\circ} \mathrm{C}$. After cooling to
A

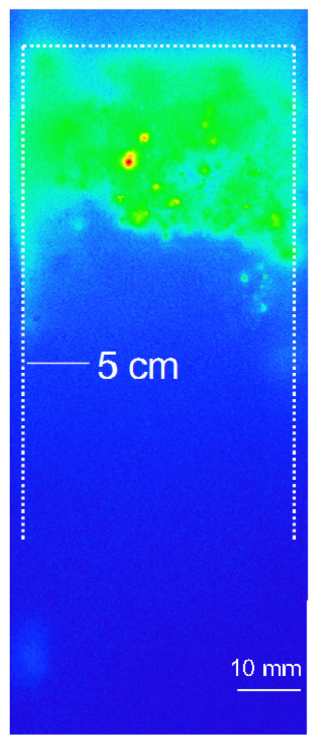

B
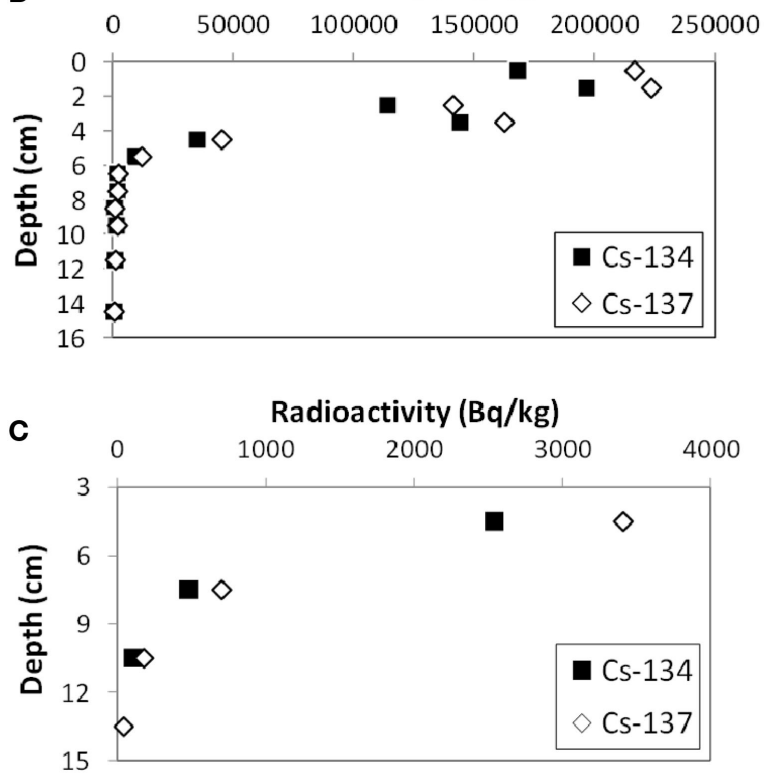

Cs-134 / Cs-137

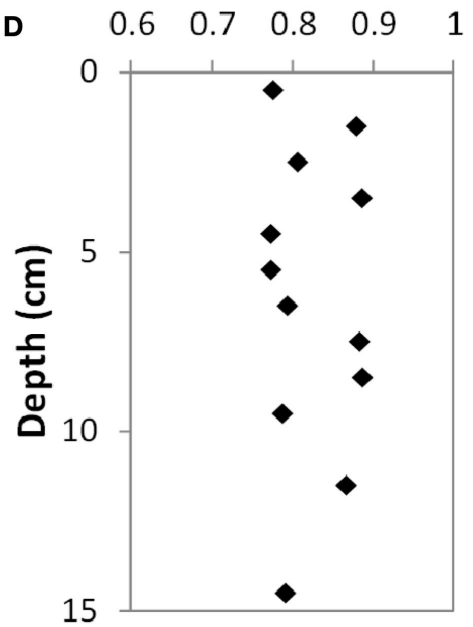

FIGURE 2 | Vertical distribution of Cs in the KOI-E core sample. (A) Autoradigraphy image of cross section of the core sample. (B) ${ }^{134}$ Cs and ${ }^{137}$ Cs radioactivity in the soil core sample. The surface of the core along the plastic liner tube was carefully decontaminated. (C) The enlarged figure of (B). (D) The radioactivity ratio of ${ }^{134} \mathrm{Cs}$ and ${ }^{137} \mathrm{Cs}$ for the vertical profile. 
room temperature, the suspension was centrifuged and filtered. The residue (residue-4) was rinsed with pure water. For the form bound to organic matter (F5), $30 \% \mathrm{H}_{2} \mathrm{O}_{2}-\mathrm{HNO}_{3}$ at $\mathrm{pH} 2.0$ was added to the residue-4, and the suspension was agitated for $2 \mathrm{~h}$ at $95 \pm 5^{\circ} \mathrm{C}$. After cooling to room temperature, the suspension was centrifuged and filtered. This extraction procedure with peroxide and nitric acid was repeated twice. Subsequently, $3.2 \mathrm{M}$ $\mathrm{NH}_{4} \mathrm{OAc}-20 \% \mathrm{HNO}_{3}$ at $\mathrm{pH}$ of 2.0 was added to the residue (residue-5a), and the suspension was agitated for $1 \mathrm{~h}$ at room temperature. The suspension was centrifuged and filtered. The residue (residue-5b) was rinsed with pure water. Insoluble residue (F6) was calculated by subtracting the sum of the radioactivities of F1-F5 from the total radioactivity.

\section{Powder X-Ray Diffraction Analysis}

Major mineral phases in the soils were determined by powder XRD (Rigaku MultiFlex) with Cu target and reflected beam monochromator. Measurement was conducted for the scan range of $3^{\circ}-63^{\circ}$ with the scanning speed of $1^{\circ} / \mathrm{min}$ of $2 \theta$ and the step angle of $0.02^{\circ}$ for bulk soil sample and elutriated soil sample $(<1 \mu \mathrm{m})$. In order to obtain the detailed structural information, the elutriated samples were measured for the focused angle ranging $3^{\circ}-13^{\circ}$ at the scan rate of $0.125^{\circ}$ or $0.25^{\circ} / \mathrm{min}$ of $2 \theta$ with the step angle of $0.02^{\circ}$. In addition, ethylene glycol treatment was also tested for the elutriated sample for the range of $3^{\circ-} 13^{\circ}$ at the scan rate of $0.125^{\circ}$ or $0.25^{\circ} \mathrm{min}^{-1}$ of $2 \theta$ with the step angle of $0.02^{\circ}$.

\section{Micro-Scale Analysis}

Individual particle analysis of the soil samples was performed using a scanning electron microscopy (SEM, SHIMADZU SS550) equipped with energy dispersive X-ray spectroscopy (EDX). Secondary electron (SE) imaging was conducted at $5 \mathrm{kV}$ of the acceleration voltage, and the EDX point analysis and mapping were completed at $25 \mathrm{kV}$. SEM wave-length dispersive X-ray spectroscopy (SEM-WDX, Hitachi High-Technologies) was also employed for searching the Cs peak in soil samples. The operating condition was $20 \mathrm{kV}$ of the acceleration voltage and $37.6 \mathrm{nA}$ of the beam current. The measurement was conducted in the range of $4213-4359 \mathrm{eV}$ at the scan speed of 1 or $2 \mathrm{eV} / \mathrm{ch}$. All samples were coated with carbon using a carbon coater (SANYU SC-701C) to make conductivity.

High-resolution TEM (HRTEM), with EDX, and high-angle annular dark-field scanning electron microscopy (HAADFSTEM) were performed using JEOL ARM200F with the acceleration voltage of $200 \mathrm{kV}$. The JEOL Analysis Station software was used to control the STEM-EDX mapping. To minimize the effect of sample drift, a drift-correction mode was used in the acquisition of the elemental map. The STEM probe size was $\sim 1.0 \mathrm{~nm}$. The collection angle of the HAADF detector was $\sim 50-110 \mathrm{mrad}$. The condenser aperture was $20 \mu \mathrm{m}$ in diameter. TEM specimens were prepared by dispersing the sample on the holey-carbon thin film supported by a $\mathrm{Cu}$ mesh grid.

\section{Cs Radioactivity Measurement}

The ${ }^{134,137}$ Cs radioactivities were measured using a gamma spectrometer equipped with a low background type Ge detector (EG\&G ORTEC Ltd GMX, relative efficiency 55.4\%) housed in the radioisotope center of Kyushu University. A spectrum analyzing software (SEIKO EG\&G Ltd. Gamma Studio) was used to determine specific isotope contributions to the radioactivity.

\section{Results and Discussion}

\section{Distribution of Cs Radioactivity in the Soil Profile}

Radioactivities of ${ }^{134} \mathrm{Cs}$ and ${ }^{137} \mathrm{Cs}$ in the soil samples are summarized in Table 1. All samples revealed both ${ }^{134} \mathrm{Cs}$ and ${ }^{137} \mathrm{Cs}$ activities $>100,000 \mathrm{~Bq} / \mathrm{kg}$, confirming the severe contamination. In particular, $2.66 \times 10^{7} \mathrm{~Bq} / \mathrm{kg}$ of ${ }^{137} \mathrm{Cs}$ was detected in KOI-W sample due to the Cs accumulation beneath a rainwater pipe. Radioactivities of the other soil samples are of a similar order of magnitude, for samples collected from the open fields.

Figure 2A shows the cross-sectional autoradiograph image of KOI-E sample. It is evident that most of the radioactive Cs is concentrated in the top $\sim 4 \mathrm{~cm}$. Slightly elevated contrast along the outer-edge facing toward the plastic tube is a result of contamination from the surface soil during the coring process. There are some hot particles (appearing as red colored spots) near surface, indicating that some particles possesses relatively higher radioactivity among the other soil components.

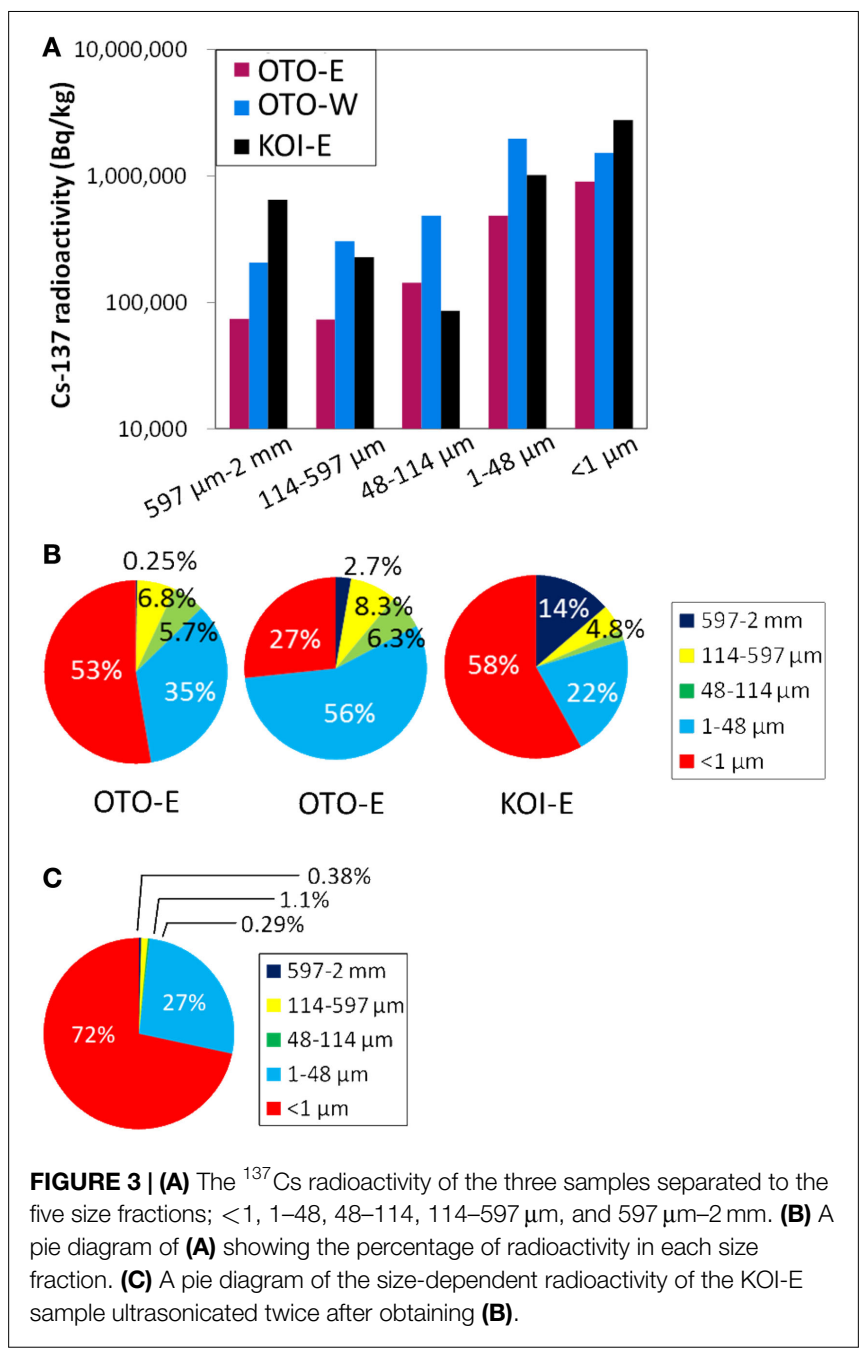



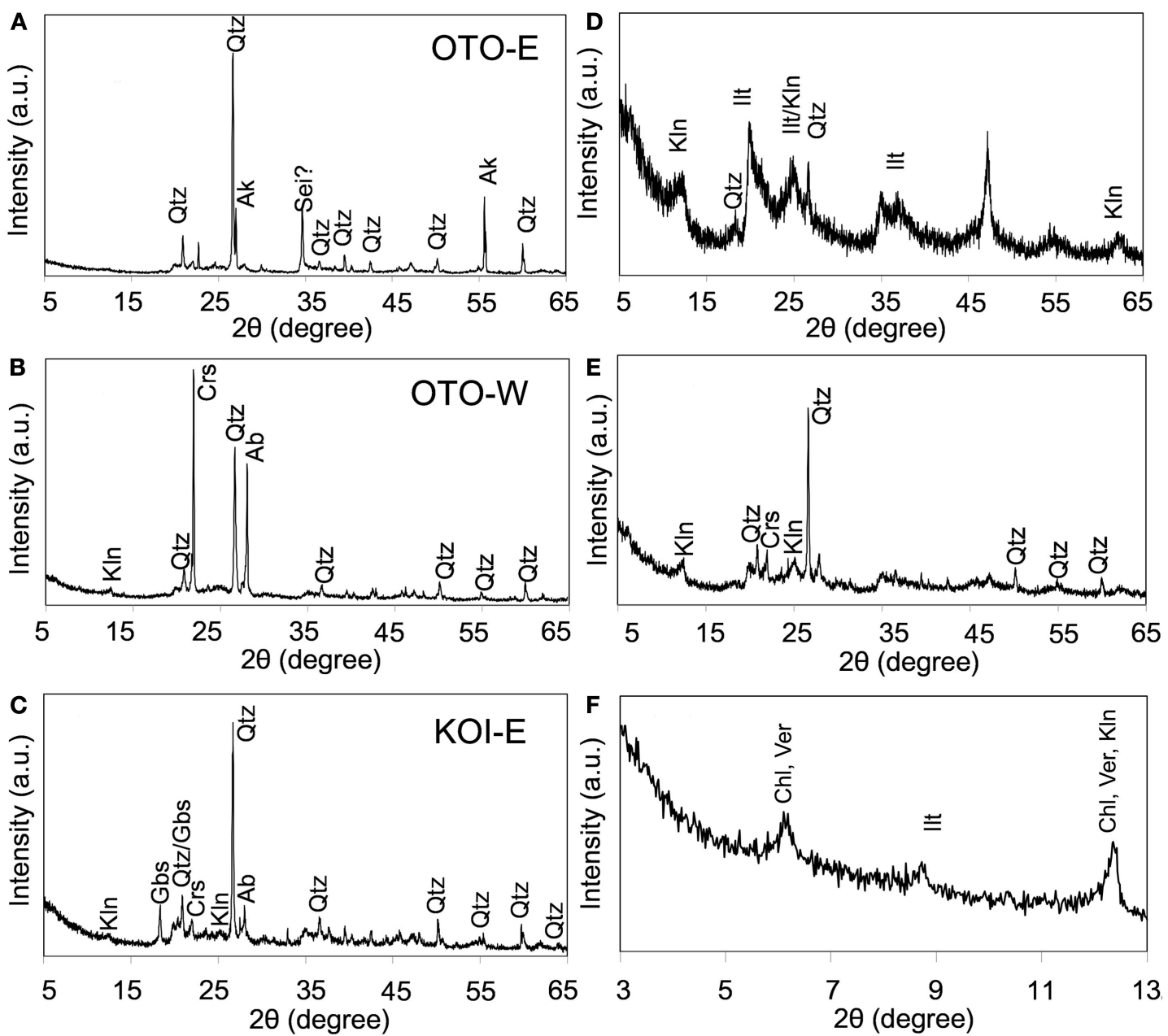

FIGURE 4 | X-ray diffraction spectrum of three soil samples: OTO-E, OTO-W, and KOI-E. (A-C) The patterns obtained from the bulk samples. (D-F) The patterns obtained from submicron-sized particle after elutriation of

the samples (A-C), respectively. Qtz, quartz; KIn, kaolinite; Crs, cristobalite; $\mathrm{Ab}$, albite; Ilt, mica and illite; Gbs, gibbsite; Crs, cristobalite; Ab, albite; Ver, vermiculite; Chl, chlorite.

Figures 2B-D shows the Cs radioactivities in the KOI-E core sample and the activity ratio of ${ }^{134} \mathrm{Cs} /{ }^{137} \mathrm{Cs}$ as a function of depth from the soil surface. The Cs contamination on the side surface of the core derived from the coring process was removed. A large fraction of the radioactive $\mathrm{Cs}$ is retained at the top of the profile (Figure 2A); ${ }^{137} \mathrm{Cs}$ radioactivity in the $0-5 \mathrm{~cm}$ interval accounts for more than $98 \%$ of the total ${ }^{137} \mathrm{Cs}$ radioactivity. These data suggest that Cs has not migrated to deeper parts of the soil profile, even 1 year after the accident. Retention of Cs in upper soil is consistent with previous studies reporting results for other Fukushima soils collected 1 month after the accident (Kato et al., 2012; Tanaka et al., 2012a). The ${ }^{134} \mathrm{Cs} /{ }^{137} \mathrm{Cs}$ activity ratios in the depth profile are almost constant, ranging 0.8-0.9 (Figure 2D), which is also consistent with the results of Kinoshita et al. (2011).

\section{Size Fraction of Cs Radioactivity in the Soils}

In order to investigate the particle size dependence of Cs radioactivity, three soils (OTO-E, OTO-W, and KOI-E) were separated into five fractions, $597 \mu \mathrm{m}-2 \mathrm{~mm}, 114-597,48-114$,
$1-48$, and $<1 \mu \mathrm{m}$ by a filtering or sedimentation method. The size-fractionated ${ }^{137} \mathrm{Cs}$ radioactivity revealed that relatively high ${ }^{137} \mathrm{Cs}$ is present in the smaller size fraction; $<1$ and $<48 \mu \mathrm{m}$, while about $10^{5} \mathrm{~Bq} / \mathrm{kg}$ of ${ }^{137} \mathrm{Cs}$ still exist in the large particle fractions: 114-597 $\mu \mathrm{m}$ and $597 \mu \mathrm{m}-2 \mathrm{~mm}$ (Figure 3A). The highest radioactivity was detected in the $<1 \mu \mathrm{m}$ fraction in OTO-E and KOI-E (Figure 3B). Specifically, the more than $3.0 \times 10^{6} \mathrm{~Bq} / \mathrm{kg}$ of ${ }^{137} \mathrm{Cs}$ in this fraction from KOI-E accounts for $58 \%$ of the total radioactivity, confirming that the greatest proportion of the radioactivity is to be found in the submicron-sized particles. Because small particles bearing ${ }^{137} \mathrm{Cs}$ can aggregate and form a larger particle, a certain portion of radioactivity derived from submicron-sized particles must also occur in the large size fractions. In order to quantify the actual Cs radioactivity of the large size fractions, the same specimen of KOI-E was sieved for the size fraction, $597 \mu \mathrm{m}-2 \mathrm{~mm}, 114-597$, and $48-114 \mu \mathrm{m}$ in Figure 3 was ultrasonicated, and the radioactivities were measured again. As shown in Figure 3C, the Cs radioactivity of the three larger size fractions decreased dramatically by an order of magnitude. This significant 


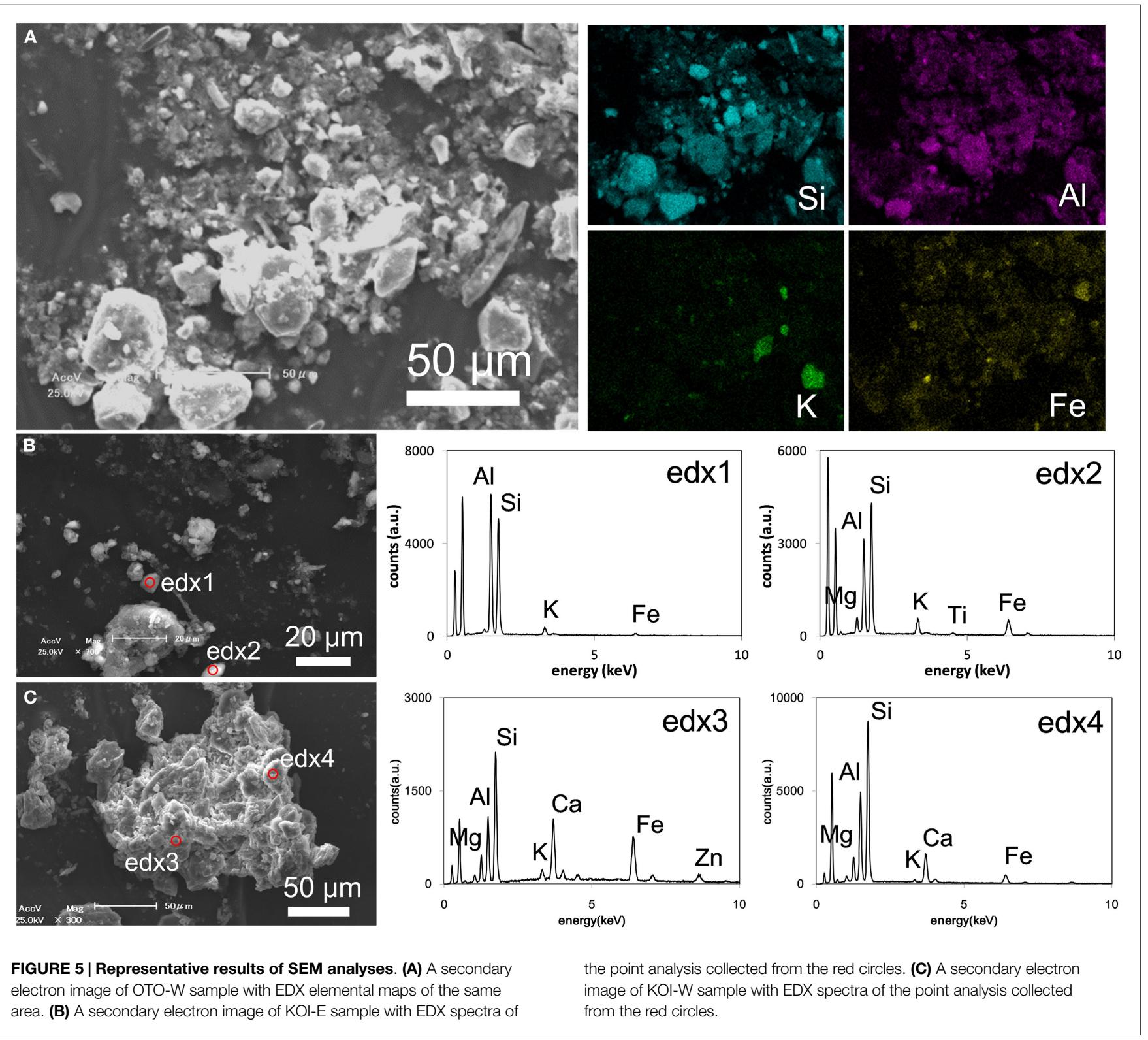

decrease of radioactivity by the ultrasonication confirms of the extensive aggregation and attachment of submicron-sized Cs particles to larger particles. The specific surface area, which is typically proportional to the number of surface adsorption site for ions, increases as the particle size decreases. However, in this case, the preferential distribution of $\mathrm{Cs}$ in the submicron-sized particle cannot be simply explained by the increased number of adsorption site for Cs. The cation exchange capacity, as determined for Fukushima soils is on the order of $1 \sim 5 \mathrm{cmol} / \mathrm{kg}$ (Iwata et al., 2012), which is eight orders of magnitude greater than the Cs concentrations. If $10^{6} \mathrm{~Bq} / \mathrm{kg}$ of ${ }^{137} \mathrm{Cs}$ occur in a soil, this corresponds to only $3 \times 10^{-2} \mathrm{ppb}$. Thus, despite the great abundance of adsorption sites in the small particle fraction, only a small number of these sites are occupied by Cs. In the case of OTO$\mathrm{W}$, the ${ }^{137} \mathrm{Cs}$ radioactivities in the bulk and submicron fraction are $1.11 \times 10^{6}$ and $1.53 \times 10^{6} \mathrm{~Bq} / \mathrm{kg}$, respectively. This difference is not consistent with the difference in the surface areas that are simply estimated based on the particle size. Thus, it appears that the very high-surface area of the small particle fraction is not entirely responsible for the high level of radioactivity in very small particle size fractions.

\section{Characterization of Soil Minerals}

Major mineral phases in the three soils, OTO-E, OTO-W, and KOI-E, were determined by XRD analysis (Figures 4A-C). Quartz was dominant in the three samples. Other mineral phases vary depending on the soils; possible akaganeite in OTO-E, kaolinite, cristobalite, and albite in OTO-W, kaolinite, gibbsite, cristobalite, and albite in KOI-E. The XRD patterns of the elutriated samples containing only submicron-sized particles in OTO-W and KOI-E revealed that the intensity of the diffraction peaks of quartz and albite decreased, while gibbsite and $7 \AA$ peaks were 
enhanced (Figures 4D,E). Slow-scan analysis of the elutriated sample of KOI-E at the low angles showed the characteristic diffraction peaks of clay minerals corresponding to 14,10 , and $7 \AA$ (Figure 4F). The possible phases based on these diffraction maxima are vermiculite, smectite, and chlorite for $14 \AA$, micas for $10 \AA$, and kaolinite for $7 \AA$.

A SEM-EDX elemental map of OTO-W sample revealed that the presence of various minerals, such as quartz, feldspar, Feoxide, and aluminosilicate (Figure 5A). It was difficult to detect Cs peak because the low concentration adsorbed on the soil minerals was below the detection limit of EDX, $\sim 0.1 \mathrm{wt} \%$. Aluminosilicates were further analyzed for their composition (Figures $5 \mathrm{~B}, \mathrm{C}$ ). The particle of edx 1 may be kaolinite or zeolite. The edx 2 represents biotite composition. The edx 3 and 4 likely indicate a mixture of biotite-vermiculite and possible Ca-carbonate. Wave-length dispersive X-ray analysis was also attempted on the sheet silicate minerals; however, Cs was not detected (data not shown).

A droplet of the elutriated suspension of OTO-W that contains submicron-sized particle was dried on a silicon wafer (Figure 6). The particles concentrated at the edge of droplet while drying due to the surface tension and arrayed as ring shape on the substrate. The autoradiograph of this sample revealed that the radioactive Cs is also distributed in the same manner as the submicron-sized particles that were confirmed in the SEM image (Figures 6A,B). The average chemical composition of these particles was $\mathrm{Si}, \mathrm{Al}$, $\mathrm{Fe}$, and little $\mathrm{K}$ (Figure 6C). The SEM analysis in conjunction with the autoradiography indicates that radioactive Cs was mainly associated with the submicron-sized aluminosilicates.

The submicron-sized particles elutriated from OTO-E were further characterized by TEM. Figures 7A-C show HAADF-STEM images of an aggregate of the submicron-sized particles. A wide area view (Figure 7A) shows numerous flakyshaped particles in the aggregate, which correspond to the texture of clay minerals like vermiculite. The flaky-shape particle appears to consist of a few domains of layered particles with different contrast (Figure 7B). Single layered particles were analyzed by EDX point analysis (edx1 and 2), revealing that the major composition is consistent with that of vermiculite (Figure 7C). Unfortunately, the Cs concentration was too low to detect by EDX. Figure 7D is the elemental maps of Figure 7A revealing the dominant occurrence of aluminosilicate with some Fe and occasional little $\mathrm{K}$. The Fe-rich and Ti-rich particles are likely Feoxide and Ti-oxides, respectively. Bright-field TEM image of the clay particles clearly shows the shape of the clay aggregates as large as a few hundred nanometer in length and several $10 \mathrm{~nm}$ in width (Figure 7E). A HRTEM image of individual particles revealed that tetrahedral-octahedral-tetrahedral layers continue only $<10$ layers with spacing of $\sim 1.0 \mathrm{~nm}$ Figure $7 \mathbf{F}$. These clays are most likely anhydrated vermiculite in conjunction with EDX analysis.

\section{Irreversible Sorption of Cs on the Surface Soils}

Figure 8A shows the results of sequential extraction for three samples: OTO-E, OTO-W, and KOI-E revealing that 3.95-21.4\% of ${ }^{137} \mathrm{Cs}$ was leached by $\left.1 \mathrm{M} \mathrm{NH} \mathrm{NHAc}_{4} \mathrm{OH} 8\right)$ solution and more than $63 \%$ of Cs was retained in the insoluble fraction. This result is consistent with the previous studies reporting that a small fraction of radiocesium in the interlayer of phyllosilicate minerals is exchangeable with $\mathrm{NH}_{4}^{+}$and a large fraction is fixed in the structure, probably along frayed edges (Evans et al., 1983; Cremer et al., 1988; McKinley et al., 2004). Because the Cs in the aerosols emitted just after the accident were predominantly water soluble, 50-90\% (Tanaka et al., 2012b), the soluble Cs was delivered by rain water and fixed in the structure of phyllosilicate minerals. Comans and Hockley (1992) studied the irreversibility of Cs sorbed onto the clay minerals and concluded that the highly selective Cs sorption site was at frayed edge sites (FES) that develop along the weathered edges of micas. Their immediate weathering products are hydrous-mica and illite. At the FES, wedge zones form, and
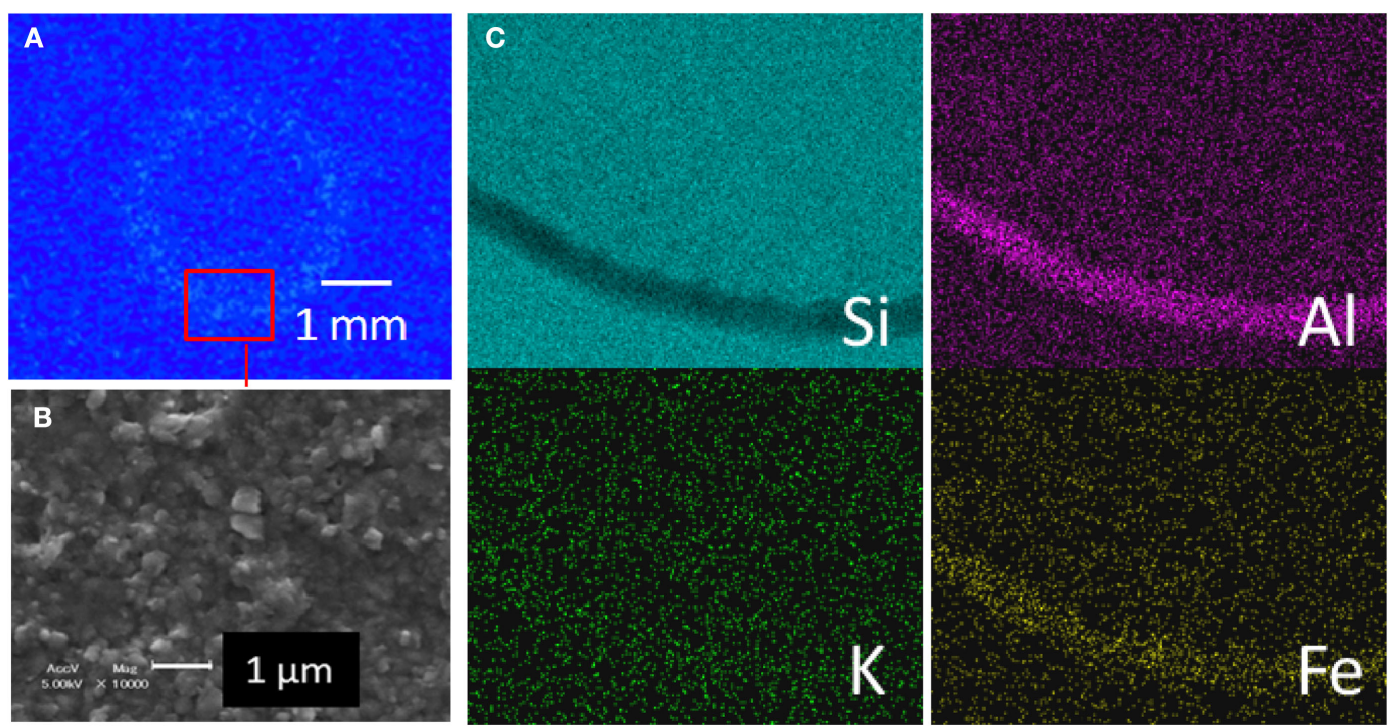

FIGURE 6 | (A) Autoradiography of a droplet of the suspended submicron-sized particles elutriated from OTO-W sample, which was dried on a smooth Si-metal plate. (B) A secondary electron image of a part of the area indicated by the red square in (A). (C) A SEM-EDX elemental map obtained from the area indicated by the red square in $\mathbf{( A )}$. 

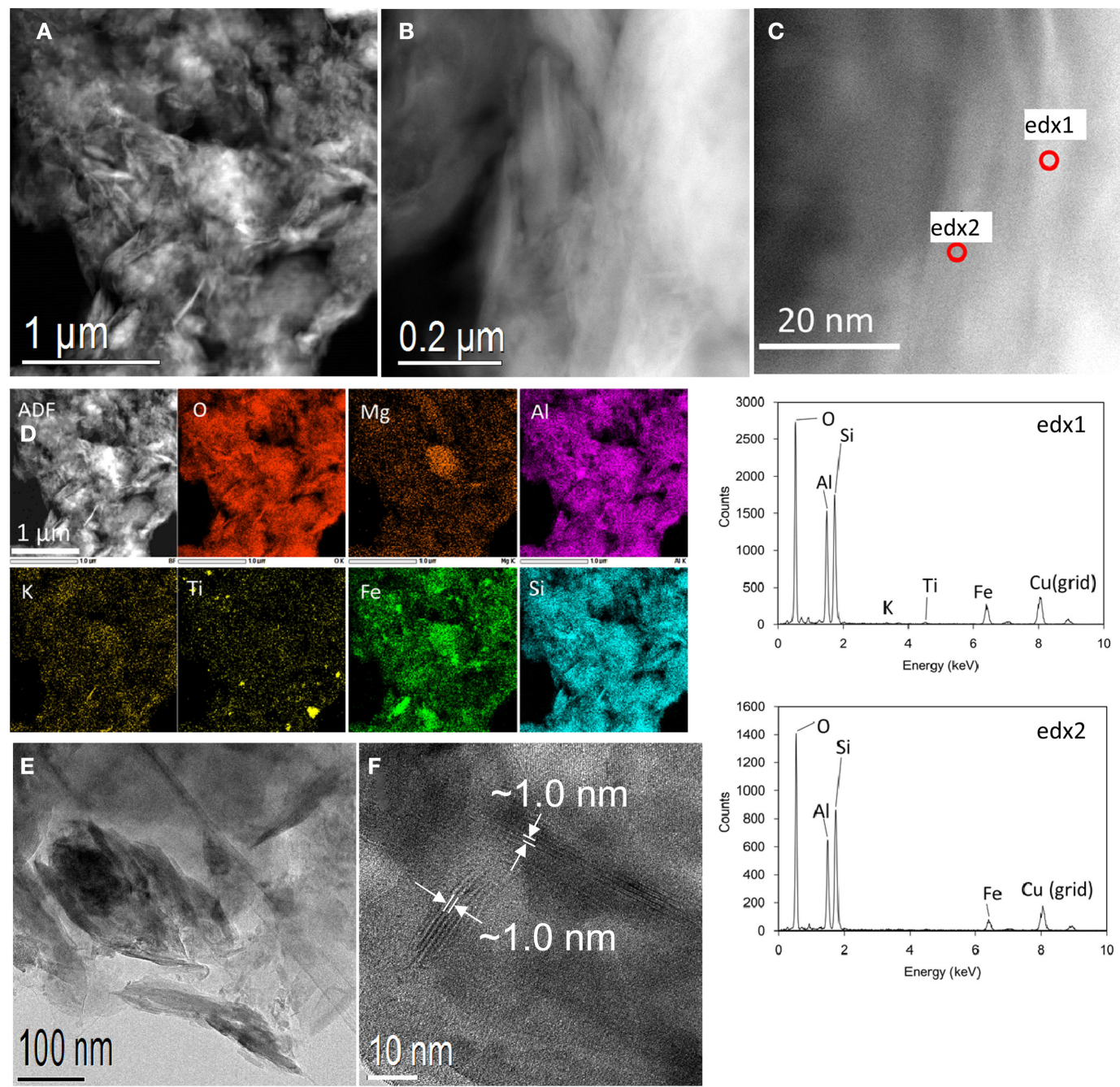

FIGURE 7 | Results of TEM analyses of the submicron-sized particles elutriated from OTO-E. (A-C) HAADF-STEM images of an aggregate of the submicron-sized particles. (A) A wide view, (B) an enlarged image of (A) showing flaky-shaped particles, and (C) a further enlarged image indicating the position of EDX point analyses. (D) Elemental maps of the area imaged in (A). (E) Bright-field TEM image of the clay particles. (F) A HRTEM image of individual particles showing the lattice spacing of $\sim 1.0 \mathrm{~nm}$. the $1.0 \mathrm{~nm}$ mica sheet opens to $1.4 \mathrm{~nm}$ vermiculite, providing a stereoselective environment for $\mathrm{Cs}^{+}$sorption (Jackson, 1963). The selectivity coefficients of Cs relative to the other cations $\left(\mathrm{Na}^{+}, \mathrm{K}^{+}\right.$, and $\left.\mathrm{Ca}^{2+}\right)$ expressed as $\log K_{\mathrm{c}},\left(K_{\mathrm{c}}=\left[A^{\mathrm{u}+}\right]\left[E_{\mathrm{CsX}}\right]^{\mathrm{u}} /\left[\mathrm{Cs}^{+}\right]^{\mathrm{u}}\left[E_{\mathrm{AXu}}\right]\right.$, where $E_{\mathrm{CsX}}$ and $E_{\mathrm{AXu}}$ represent adsorption density divided by CEC for Cs and cation A) estimated to be up to $\sim 6.76$ based on the ion exchange models (Bradbury and Baeyens, 2000; Zachara et al., 2002), stating the high affinity of Cs with sheet aluminosilicate. Hence, in the soils at Fukushima, similar Cs-binding mechanisms may play a role in fixing Cs onto aluminosilicate sheet structures; hence, the release of radioactive Cs from soil minerals should be minimal. A limited amount of Cs can be released by an ion exchange process, but it is expected that the released soluble Cs will be immediately adsorbed onto aluminosilicate minerals with a sheet structure, that is clays.

Sequential extraction was also conducted for the submicronsized particle elutriated from OTO-W sample (Figure 8B).
The chemical form of Cs in the submicron-sized particle fraction is similar to that in the bulk, although the major mineral constituents were different from that of the bulk. Quartz and feldspar were major minerals in bulk; whereas, clays and micalike minerals were dominant in the submicron-sized fraction. The results of sequential extraction indicate that $\mathrm{Cs}$ is predominantly adsorbed onto the clays in the bulk sample, as well as in the submicron-sized fraction, because the number of adsorption site on the sheet structure of the aluminosilicate clays is much greater than the amount of Cs available. The sequential extraction for Cs in Fukushima soils probably represents the chemical form of Cs adsorbed on the submicron-sized sheet alumino silicate.

\section{Role of Submicron-Sized Particles as Cs Carrier}

In general, submicron-sized particles in aquifer behave as colloids, which have unique properties in the subsurface. Colloidfacilitated transport of toxic elements is typically ascribed to 

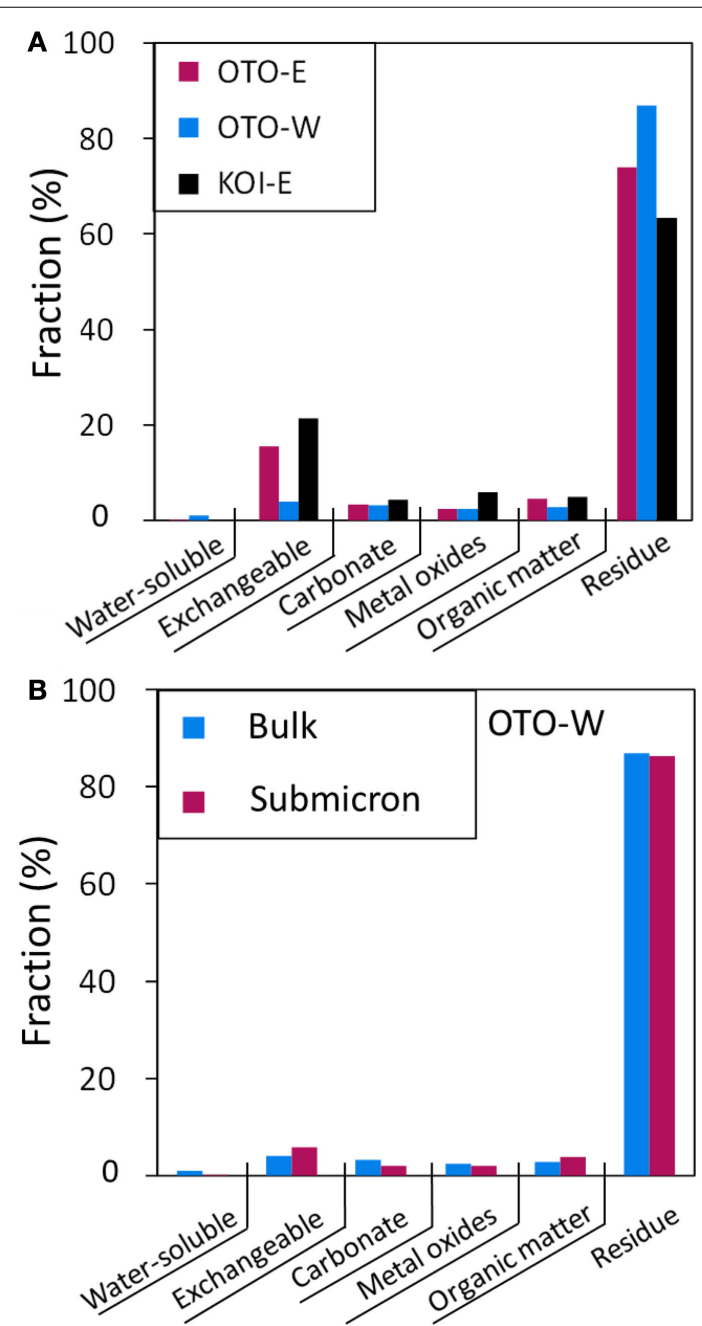

FIGURE 8 | (A) Fraction of the Cs chemical form in three soil samples determined by a sequential extraction. (B) Fraction of Cs chemical form in the submicron-sized particle elutriated from OTO-W as compared to that in the bulk OTO-W soil.

hydrodynamic chromatographic effects (McCarthy and Zachara, 1989; Kim, 1991). In the heavily contaminated soils of the present study, the ${ }^{137} \mathrm{Cs}$ radioactivity was $\sim 3,000,000 \mathrm{~Bq} / \mathrm{kg}$ on the colloids and these submicron-sized particles can potentially contribute to the migration of radioactive Cs as pseudo-colloids. Short range migration of Cs particles might occur only within top of the profile as reported in Ohnuki and Tanaka (1989) and Tanaka and Ohnuki (1996). However, the particles that have sorbed the radioactive Cs remain within the top $\sim 5 \mathrm{~cm}$ of vertical profile (Figure 2 ), and the amount of radioactivity in groundwater collected from a well at Iitate village was below the detection limit (data not shown). This suggests that Cs migration through vadose zone to groundwater

\section{References}

Bradbury, M., and Baeyens, B. (2000). A generalised sorption model for the concentration dependent uptake of caesium by argillaceous rocks. J. Contam. Hydrol. 42, 141-163. doi:10.1016/S0169-7722(99)00094-7 did not occur for the period from March 2011 to March 2012. As shown in the results above, a large fraction of Cs was adsorbed onto the submicron-sized aluminosilicates with sheet structures, that is clays, and although they are potentially mobile, their movement has been limited because the small particles have aggregated due to the fact that the $\mathrm{pH}$ of the ground water is near neutral and the point of zero charge (PZC) of sheet aluminosilicate is $\sim 6-7$ [based on the PZC of biotite as reported in Goldberg et al. (2012)]. Formation of aggregates has prevented the Cs particles from being transported vertically through the soils due to a filtering effect. Rather, the aggregates of small Cs particles currently migrate through surface water. Indeed, $90-98 \%$ of the radioactive Cs is transported as suspended fine particle from hydrographic basin to rivers, which is supposed to be a major dose contributor of the Cs flux to the ocean: $200-500 \mathrm{GBq} /$ year for 5 years after the accident (JAEA, 2013). As a consequence, transport of Cs particle aggregates through surface water should have been responsible for the removal of Cs radioactivity of surface environment to the lower level than that simply calculated by radioactive decay of ${ }^{134} \mathrm{Cs}$.

\section{Conclusion}

The submicron-sized particles in the contaminated soils near Fukushima are mainly composed of aluminosilicates with sheet structures, clays. These clays, mica, vermiculite, chlorite, and smectite, are responsible for adsorbing more than $70 \%$ of the total Cs in the soil profile. The adsorbed Cs does not desorb easily, probably because of strong binding at FES of the clays (Iwata et al., 2012). Aggregation of the very small clay particles into larger particles has further limited their migration downward through the soil profile; hence, most of the radioactivity remains within the top $5 \mathrm{~cm}$ of the soil profile. Currently, the mobility of the aggregates of submicron-sized sheet aluminosilicate in the surface environment is a key factor controlling the Cs migration in Fukushima. Still, the aggregates can be potentially transported as suspended colloids in case of deflocculation in a specific geochemical condition of ground water, and this should be considered in transport models that predict future migration of Cs in these highly contaminated regions.

\section{Acknowledgments}

This study is partially supported by JST Initiatives for Atomic Energy Basic and Generic Strategic Research and by the Science Grant of the Ministry of Education, Science and Culture. SU is also supported by ESPEC Foundation for Global Environment Research and Technology (Charitable Trust) (ESPEC Prize for the Encouragement of Environmental Studies). SU is also grateful to Dr. Watanabe for her assistance on SEM and XRD analyses at the Center of Advanced Instrumental Analysis, Kyushu University.

Comans, R., and Hockley, D. (1992). Kinetics of cesium sorption on illite. Geochim. Cosmochim. Acta 56, 1157-1164. doi:10.1016/0016-7037(92)90053-L

Cremer, A., Elsen, A., De Preter, P., and Maes, A. (1988). Quantitative analysis of radiocaesium retention in soils. Nature 335, 247-249. doi:10.1038/ $335247 \mathrm{a} 0$ 
Evans, D., Alberts, J., and Clark, R. (1983). Reversible ion-exchange fixation of cesium-137 leading to mobilization from reservoir sediments. Geochim. Cosmochim. Acta 47, 1041-1049. doi:10.1016/0016-7037(83)90234-X

Goldberg, S. R., Lebron, I., Seaman, J. C., and Suarez, D. L. (2012). "Chapter 15: soil colloidal behavior," in Handbook of Soil Sciences Properties and Processes, 2nd Edn, eds P. M. Huang, Y. Li, and M. E. Sumner (Boca Raton, FL: CRC Press, Taylor and Francis Group), 15-1-15-39.

Hou, X., Fogh, C., Kucera, J., Andersson, K., Dahlgaard, H., and Nielsen, S. (2003). Iodine-129 and caesium-137 in chernobyl contaminated soil and their chemical fractionation. Sci. Total Environ. 308, 97-109. doi:10.1016/S0048-9697(02) 00546-6

Iwata, H., Shiotsu, H., Kaneko, M., and Utsunomiya, S. (2012). "Nuclear accidents in Fukushima, Japan and exploration of effective decontaminant for the ${ }^{137} \mathrm{Cs}$ contaminated soils," in Advances in Nuclear Fuel, ed. T. R. Shripad (Rijeka: Intech), 123-142.

Jackson, M. L. (1963). Interlayering of expansible layer silicates in soils by chemical weathering. Clays Clay Miner. 11, 29-46. doi:10.1346/CCMN.1962.0110104

JAEA. (2013). Available at: http://fukushima.jaea.go.jp/initiatives/cat03/entry06. html

Kaneyasu, N., Ohashi, H., Suzuki, F., Okuda, T., and Ikemori, F. (2012). Sulfate aerosol as a potential transport medium of radiocesium from the Fukushima nuclear accident. Environ. Sci. Technol. 46, 5720-5726. doi:10.1021/ es204667h

Kato, H., Onda, Y., and Teramage, M. (2012). Depth distribution of Cs-137, Cs-134, and I-131 in soil profile after Fukushima Dai-Ichi nuclear power plant accident. J. Environ. Radioact. 111, 59-64. doi:10.1016/j.jenvrad.2011.10.003

Kim, J. I. (1991). Actinide colloid generation in groundwater. Radiochim. Acta $52 / 53,71-81$.

Kinoshita, N., Sueki, K., Sasa, K., Kitagawa, J., Ikarashi, S., Nishimura, T., et al. (2011). Assessment of individual radionuclide distributions from the Fukushima nuclear accident covering central-east Japan. Proc. Natl. Acad. Sci. U.S.A. 108, 19526-19529. doi:10.1073/pnas.1111724108

Koarashi, J., Atarashi-Andoh, M., Matsunaga, T., Sato, T., Nagao, S., and Nagai, H. (2012). Factors affecting vertical distribution of Fukushima accident-derived radiocesium in soil under different land-use conditions. Sci. Total Environ. 431, 392-401. doi:10.1016/j.scitotenv.2012.05.041

Kozai, N., Ohnuki, T., Arisaka, M., Watanabe, M., Sakamoto, F., Yamasaki, S., et al. (2012). Chemical states of fallout radioactive Cs in the soils deposited at Fukushima Daiichi nuclear power plant accident. J. Nucl. Sci. Technol. 49, 473-478. doi:10.1080/00223131.2012.677131

Matsunaga, T., Koarashi, J., Atarashi-Andoh, M., Nagao, S., Sato, T., and Nagai, H. (2013). Comparison of the vertical distributions of Fukushima nuclear accident radiocesium in soil before and after the first rainy season, with physicochemical and mineralogical interpretations. Sci. Total Environ. 447, 301-314. doi:10.1016/ j.scitotenv.2012.12.087

McCarthy, J., and Zachara, J. (1989). Subsurface transport of contaminants - mobile colloids in the subsurface environment may alter the transport of contaminants. Environ. Sci. Technol. 23, 496-502. doi:10.1021/es00063a602

McKinley, J., Zachara, J., Heald, S., Dohnalkova, A., Newville, M., and Sutton, S. (2004). Microscale distribution of cesium sorbed to biotite and muscovite. Environ. Sci. Technol. 38, 1017-1023. doi:10.1021/es034569m

MEXT. (2013). Available at: http://radioactivity.nsr.go.jp/ja/contents/7000/6749/ 24/191_258_0301_18.pdf

Morino, Y., Ohara, T., Watanabe, M., Hayashi, S., and Nishizawa, M. (2013). Episode analysis of deposition of radiocesium from the Fukushima Daiichi nuclear power plant accident. Environ. Sci. Technol. 47, 2314-2322. doi:10.1021/ es304620x

Mukai, H., Hatta, T., Kitazawa, H., Yamada, H., Yaita, T., and Kogure, T. (2014). Speciation of radioactive soil particles in the Fukushima contaminated area by IP autoradiography and microanalyses. Environ. Sci. Technol. 48, 13053-13059. doi:10.1021/es502849e

Nakao, A., Ogasawara, S., Sano, O., Ito, T., and Yanai, J. (2014). Radiocesium sorption in relation to clay mineralogy of paddy soils in Fukushima, Japan. Sci. Total Environ. 46, 523-529. doi:10.1016/j.scitotenv.2013.08.062

Ohnuki, T., and Kozai, N. (2013). Adsorption behavior of radioactive cesium by non-mica minerals. J. Nucl. Sci. Technol. 50, 369-375. doi:10.1080/00223131. 2013.773164

Ohnuki, T., and Tanaka, T. (1989). Migration of radionuclides controlled by several different migration mechanisms through a sandy soil layer. Health Phys. 56, 47-53. doi:10.1097/00004032-198901000-00004

Steinhauser, G., Brandl, A., and Johnson, T. E. (2014). Comparison of the chernobyl and Fukushima nuclear accidents: a review of the environmental impacts. Sci. Total Environ. 47, 800-817. doi:10.1016/j.scitotenv.2013.10.029

Tanaka, K., Takahashi, Y., Sakaguchi, A., Umeo, M., Hayakawa, S., Tanida, H., et al. (2012a). Vertical profiles of iodine-131 and cesium-137 in soils in Fukushima prefecture related to the Fukushima Daiichi nuclear power station accident. Geochem. J. 46, 73-76. doi:10.2343/geochemj.1.0137

Tanaka, K., Sakaguchi, A., Kanai, Y., Tsuruta, H., Shinohara, A., and Takahashi, Y. (2012b). Heterogeneous distribution of radiocesium in aerosols, soil and particulate matters emitted by the Fukushima Daiichi nuclear power plant accident: retention of micro-scale heterogeneity during the migration of radiocesium from the air into ground and river systems. J. Radioanal. Nucl. Chem. 295, 1927-1937. doi:10.1007/s10967-012-2160-9

Tanaka, T., and Ohnuki, T. (1996). Colloidal migration behavior of radionuclides sorbed on mobile fine soil particles through a sand layer. J. Nucl. Sci. Technol. 33 , 62-68. doi:10.1080/18811248.1996.9731862

Tessier, A., Campbell, P. G. C., and Bisson, M. (1979). Sequential extraction procedure for the speciation of particulate trace metals. Anal. Chem. 51, 844-851. doi:10.1021/ac50043a017

Yoshida, N., and Takahashi, Y. (2012). Land-surface contamination by radionuclides from the Fukushima Daiichi nuclear power plant accident. Elements 8, 201-206. doi:10.2113/gselements.8.3.201

Zachara, J., Smith, S., Liu, C., McKinley, J., Serne, R., and Gassman, P. (2002). Sorption of $\mathrm{Cs}^{+}$to micaceous subsurface sediments from the Hanford Site, USA. Geochim. Cosmochim. Acta 66, 193-211. doi:10.1016/S0016-7037(01) 00759- 1

Conflict of Interest Statement: The authors declare that the research was conducted in the absence of any commercial or financial relationships that could be construed as a potential conflict of interest.

Copyright (c) 2015 Kaneko, Iwata, Shiotsu, Masaki, Kawamoto, Yamasaki, Nakamatsu, Imoto, Furuki, Ochiai, Nanba, Ohnuki, Ewing and Utsunomiya. This is an open-access article distributed under the terms of the Creative Commons Attribution License (CC BY). The use, distribution or reproduction in other forums is permitted, provided the original author(s) or licensor are credited and that the original publication in this journal is cited, in accordance with accepted academic practice. No use, distribution or reproduction is permitted which does not comply with these terms. 